University of Nebraska - Lincoln

DigitalCommons@University of Nebraska - Lincoln

Publications, Agencies and Staff of the U.S.

Department of Commerce

U.S. Department of Commerce

1989

\title{
EFFECTS OF TRACK STRUCTURE ON NEUTRON \\ MICRODOSIMETRY AND NANODOSIMETRY
}

\author{
Randall S. Caswell \\ National Institute of Standards and Technology
}

Follow this and additional works at: https://digitalcommons.unl.edu/usdeptcommercepub

Part of the Environmental Sciences Commons

Caswell, Randall S., "EFFECTS OF TRACK STRUCTURE ON NEUTRON MICRODOSIMETRY AND NANODOSIMETRY" (1989). Publications, Agencies and Staff of the U.S. Department of Commerce. 207. https://digitalcommons.unl.edu/usdeptcommercepub/207

This Article is brought to you for free and open access by the U.S. Department of Commerce at DigitalCommons@University of Nebraska - Lincoln. It has been accepted for inclusion in Publications, Agencies and Staff of the U.S. Department of Commerce by an authorized administrator of DigitalCommons@University of Nebraska - Lincoln. 


\title{
EFFECTS OF TRACK STRUCTURE ON NEUTRON MICRODOSIMETRY AND NANODOSIMETRY
}

\author{
Randall S. Caswell and J. Joseph Coyne \\ National Institute of Standards and Technology (formerly National Bureau of Standards), \\ Gaithersburg, MD 20899, U.S.A.
}

(Received 17 January 1989)

\begin{abstract}
The process by which neutrons transfer their energy to tissue is described, including the deposition of energy by secondary charged particle tracks in small sites; that is, microdosimetry and nanodosimetry. Three approximations are considered in order of increasing amounts of information on track structure: the continuous slowing-down approximation (CSDA), the straggling approximation, and the delta-ray approximation. Although simple approximations sometimes work quite well, much work remains to be done for very small sites, and for the inclusion of the effects of "passers" in the calculations.
\end{abstract}

\section{INTRODUCTION}

NEUTRONS interact with tissue or tissue-like materials through a two-stage process: (1) the neutrons interact with the nuclei of the medium generating secondary charged particles, chiefly $p, \alpha$, and $\mathrm{C}, \mathrm{N}$, and $\mathrm{O}$ recoil nuclei; and (2) the secondary charged particles traverse the medium, losing energy (slowing down) and interacting with the atoms of the medium chiefly through ejection of secondary electrons, ionizations and excitations, the description of which is the subject of track physics. The spectrum of energies with which the secondary particles are generated by the nuclear reactions we call the initial spectrum (an example for $2-\mathrm{MeV}$ neutrons is given in Fig. 1). The average spectrum of the particles in the medium, which is the spectrum at the biologically-sensitive sites in the medium, we call the slowing-down spectrum (Caswell and Coyne, 1976; ICRU, 1983). An example of a slowing-down spectrum for $14.5-\mathrm{MeV}$ neutrons is given in Fig. 2. These spectra are useful in a number of applications other than microdosimetry, for example, for the application of the delta-ray model of cellular inactivation to neutrons (Katz and Sharma, 1973), see Fig. 3.

In microdosimetry we are concerned with the statistical variations in the absorption of energy in small sites of the order of micrometers $(\mu \mathrm{m})$, whereas in nanodosimetry the site size is smaller, nanometers (nm), comparable to such structures as the DNA helix. Many questions arise and are being studied concerning the relation between the distributions and parameters of microdosimetry and nanodosimetry and the resulting biological effect (Goodhead, 1982; Rossi, 1985). These important questions will not be discussed further here.

\section{CONSIDERATIONS OF GEOMETRY AND PHYSICS}

For simplicity, the sensitive site is often taken as spherical, although cylindrical and ellipsoidal sites have been considered. Since the tracks of the heavy charged particles can usually be considered as straight, an analysis in terms of five kinds of track interactions with the site (or "cavity" due to the relation of this problem with cavity theory) has been useful. These interactions or tracks are called "insiders", "starters", "stoppers", "crossers", and "passers" (although the term "toucher" has also been used for the final category, see Fig. 4). If the cavity size is large compared to the particle range, "insiders" are the dominant events. If the cavity is small, "crossers" are the dominant tracks (Bragg-Gray condition). If the cavity is extremely small, few tracks will go directly through the cavity, and "passers"; tracks where the secondary electrons enter the cavity even though the main track is outside, dominate.

\section{Continuous slowing-down approximation (CSDA)}

In this approximation, the only feature of track physics that is considered is the stopping power or LET $_{\infty}$, which is the average rate of energy loss of the charged particle at a given energy. Using only the geometry of the cavity, as expressed in the chordlength distribution and the stopping power, we can calculate the lineal energy or " $y$ " spectrum for a given diameter cavity (see Fig. 5). Note in this figure that the major peak in the spectrum at around $10 \mathrm{keV} / \mu \mathrm{m}$, which is due to protons, is higher and narrower in the calculation than in the experimental measurements, an effect which we will demonstrate is 


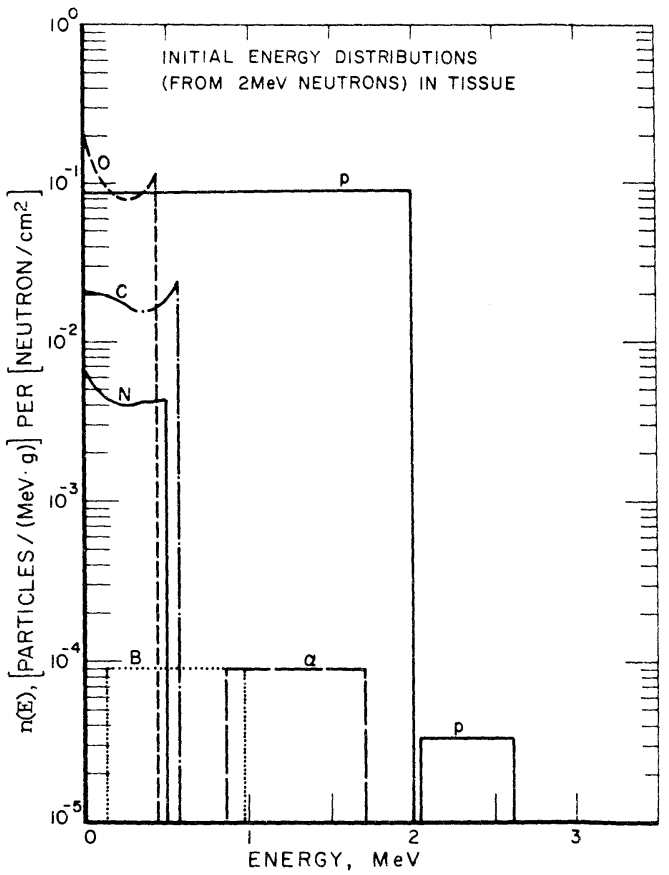

Fig. 1. Initial energy distributions of secondary charged particles generated by $2-\mathrm{MeV}$ neutrons in 4-element tissue. Note the flat proton distribution. Interactions involved at this energy are chiefly elastic scattering. At higher neutron energies the situation becomes much more complex, many nuclear reactions taking place.

due to neglect of straggling. Nevertheless, the very simple CSDA approximation usually does reasonably well in predicting the experimental data. At a low

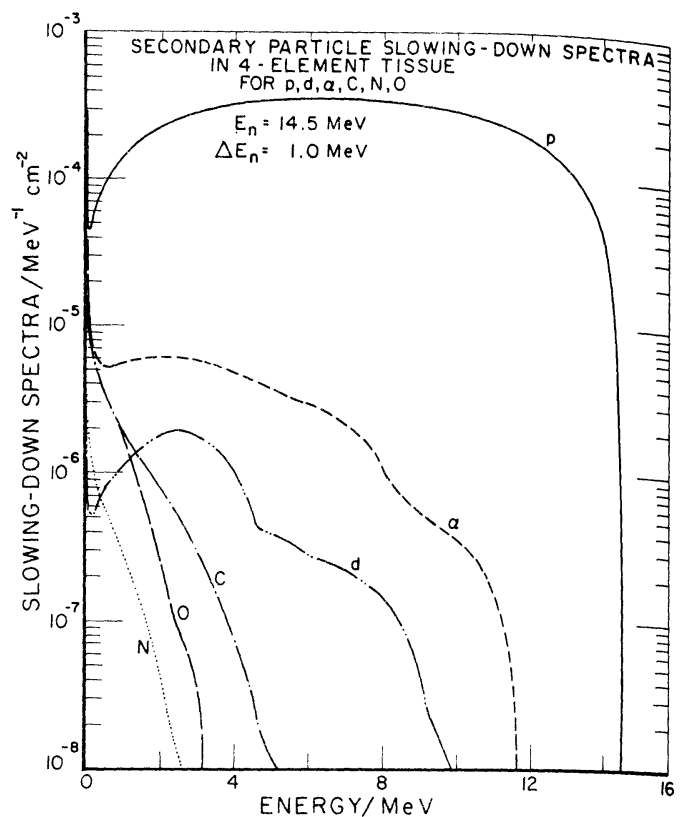

FIG. 2. Slowing-down spectra of secondary particles generated by $14.5-\mathrm{MeV}$ neutrons in 4-element tissue. The width of the energy bin centered at $14.5 \mathrm{MeV}$ is $1 \mathrm{MeV}$. Note the strong dominance of the protons over the other charged particles in the slowing-down spectra, in part because the slowing-down spectrum contains a factor inversely proportional to the stopping power.

neutron energy, $144 \mathrm{keV}$, the CSDA approximation agrees well with experimental data (Fig. 6). The proton ranges are comparable to the cavity diameter.

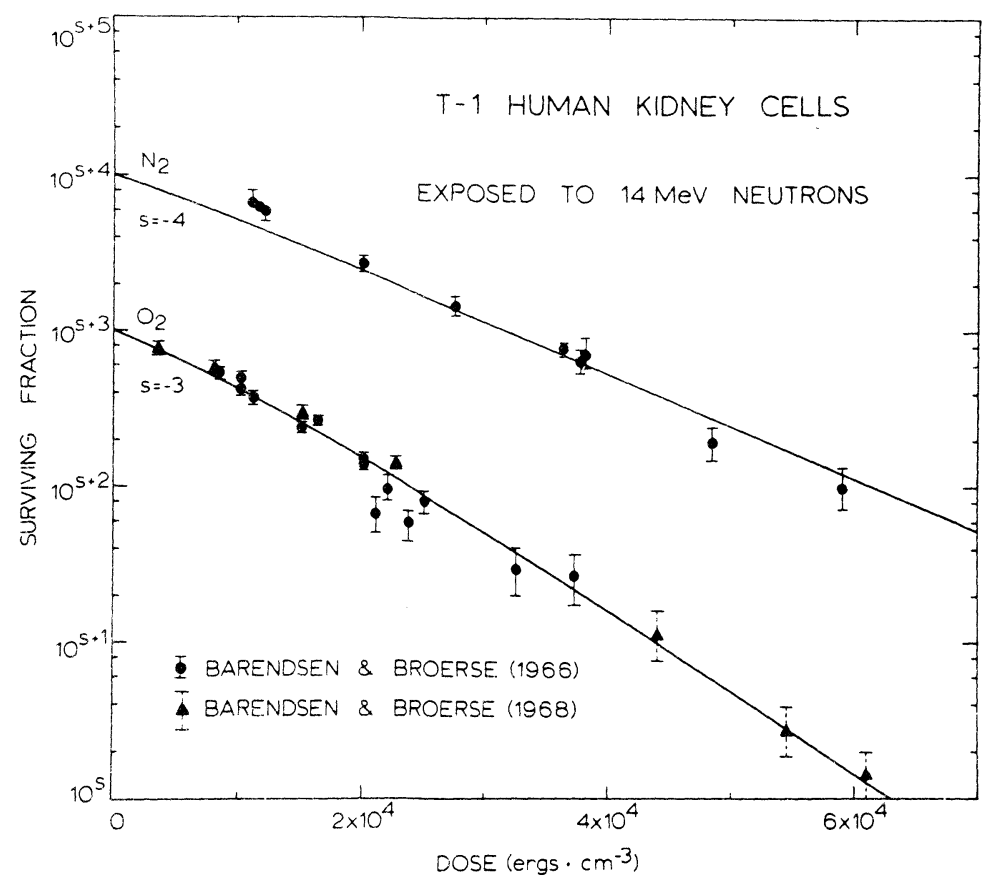

Fig. 3. Survival curves of $T-1$ human kidney cells irradiated with $14-\mathrm{MeV}$ neutrons under oxygenated and anoxic conditions as measured by Barendsen and Broerse $(1966,1968)$ compared to the calculations of Katz and Sharma (1973). The calculations used the initial spectra for secondary charged particles of Caswell and Coyne (1972). 


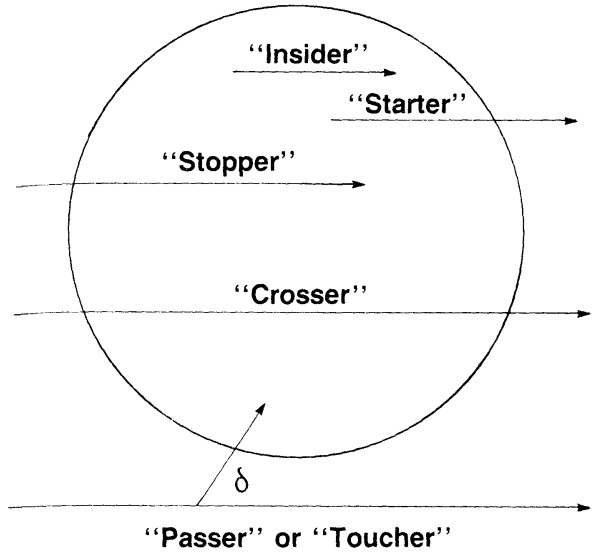

Fig. 4. Classification of track interactions with a sensitive biological site or cavity. As the site size becomes smaller, "crossers" and "passers" or "touchers" become the most important events.

\section{Straggling approximation}

In the straggling approximation we consider the energy loss of a particular track over a chord length to be equal to the energy deposition in the cavity for that track, with no explicit consideration of delta-ray effects. In Fig. 7(a) we consider two particle tracks intercepting equal chord lengths in the cavity. In CSDA the energy depositions for particles, say protons, entering the cavity with the same energies would be equal for the two tracks, since the average stopping powers along the two tracks have the same variation. In the straggling approximation the energy depositions would be different for the upper and lower tracks as given by the particular stochastic energy losses of the two tracks.

Another consideration in these calculations we call "energy balance". For example, in a medium with uniform neutron fluence and spectrum over the region considered, the kerma (charged particle kinetic energy generated) must equal the absorbed dose, $K=D$. Therefore,

$$
\int_{0}^{E_{\max }} E n(E) \mathrm{d} E=\frac{1}{V} \int f_{1}(\epsilon) \epsilon \mathrm{d} \epsilon
$$

where $n(E)$ is the initial spectrum of particles of energy $E$, and $f_{1}(\epsilon)$ is the distribution of energy depositions $\epsilon$ occurring in a cavity of volume $V$ (assuming unit-density tissue). Comparison of these two integrals serves as a check on the numerical calculation. In the case of CSDA, condition (1) should be automatically satisfied. In the straggling approximation, using available distributions such as Landau and Vavilov, condition (1) is generally not satisfied. Using the Landau distribution, unless truncated, the right-hand integral in (1) becomes infinite. In the straggling approximation, one can use a triangular-shaped straggling function which is not quite correct but retains energy balance, or use truncated distributions.

\section{Delta rays and straggling}

In Fig. 7(b) are shown schematically two equal chord length tracks with emission of a delta ray from each track. The energy deposition $\epsilon=\epsilon_{1}-\epsilon_{2}$, where

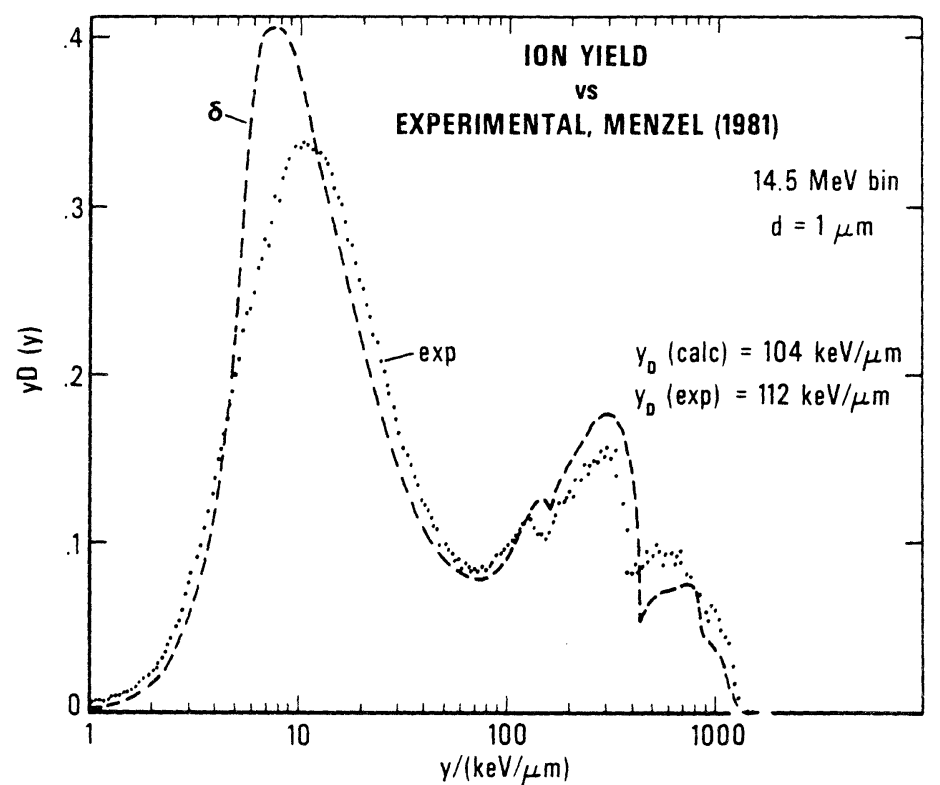

Fig. 5. Comparison of $14.5-\mathrm{MeV}$ ion yield calculation with the experimental results at $15.1-\mathrm{MeV}$ neutron energy, shown as Menzel (1981), finally published as Coyne et al. (1981). The ion yield calculation is more appropriate for comparison with experiment since the tissue-equivalent proportional counter pulse height is proportional to ionization. 


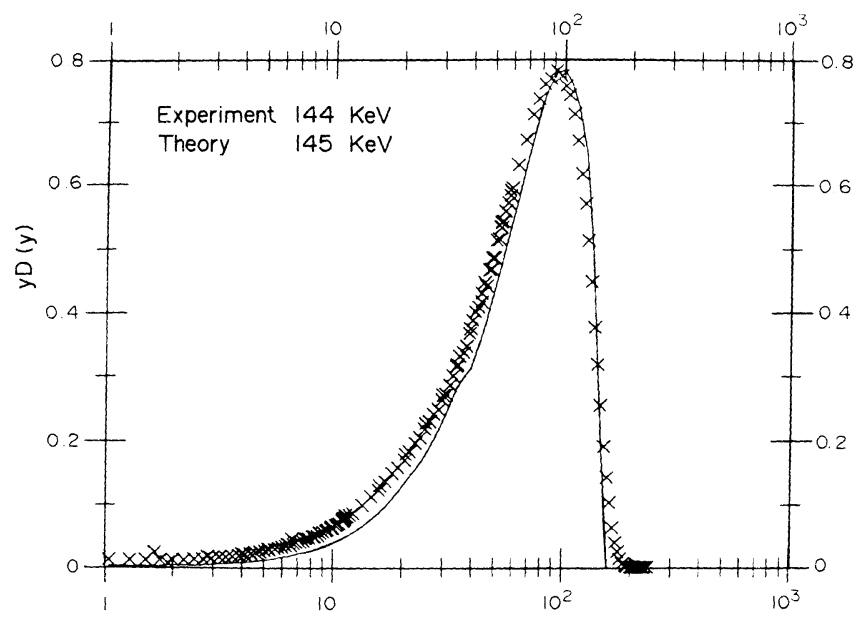

Fig. 6. Comparison of experimental microdosimetry spectrum measurements for $144-\mathrm{keV}$ reactor beam filtered neutrons with an analytic CSDA calculation for 145-keV neutrons (Caswell et al., 1986).

$\epsilon_{1}$ is the energy lost by the proton, and $-\epsilon_{2}$ is the energy carried out of the sphere by secondary electrons. The upper track in Fig. 7(b) has a non-zero $\epsilon_{2}$. For the lower track, $\epsilon_{2}$ is zero. Energy balance often does not hold for a single track. The delta-ray energy lost by tracks crossing the cavity is balanced by deposition of energy by delta rays entering the cavity from passers.

\section{MICRODOSIMETRY CALCULATIONS INCLUDING STRAGGLING}

A comparison of a CSDA calculation versus a calculation including straggling is given in Fig. 8 for 14.1-MeV neutrons where curve $B$ is the CSDA calculation, and curve $A$ is the straggled distribution using a triangular straggling distribution for the protons. By comparison with Fig. 5 we see that the straggled distribution for the proton peak is much closer to the experimental results.

In Fig. 9, a Monte Carlo calculation including proton straggling for $2.1 \mathrm{-MeV}$ neutrons is compared to an experimental measurement for $2.18-\mathrm{MeV}$ neutrons. The straggling function assumed is a Landau distribution. We see an overall satisfactory agreement, with the widths of the rather broad proton peak between about 20 and $150 \mathrm{keV} / \mu \mathrm{m}$ being about equal in calculation and experiment. The experimental points below about $8 \mathrm{keV} / \mu \mathrm{m}$ are due to photon contamination in the experiment not considered in the calculation. The points and curve above $150 \mathrm{keV} / \mu \mathrm{m}$ are due to heavy particle recoils. The difference between experiment and calculation for the heavy particles is due partly to the effect of an increased $W$, average energy per ion pair, in the experiment not compensated for in this calculation, and partly due to poor statistics in the experiment at these values of $y$.

\section{CALCULATIONS IN THE DELTA-RAY APPROXIMATION (NEGLECTING PASSERS)}

In the calculations discussed here "crossers" are the dominant event. We have used the lognormal distributions for protons of Wilson et al. (1988) which are fitted to the Monte Carlo results including delta rays of their MOCA14 code. Their distributions have been normalized slightly to give energy balance for a single track (average of the distribution is the CSDA energy loss). In Fig. 10 the dashed line is the CSDA result for protons for a $1 \mu \mathrm{m}$ cavity diameter. Heavier particles, not shown, are as in Figure 8. The solid line is the delta-ray approximation result. Note that the
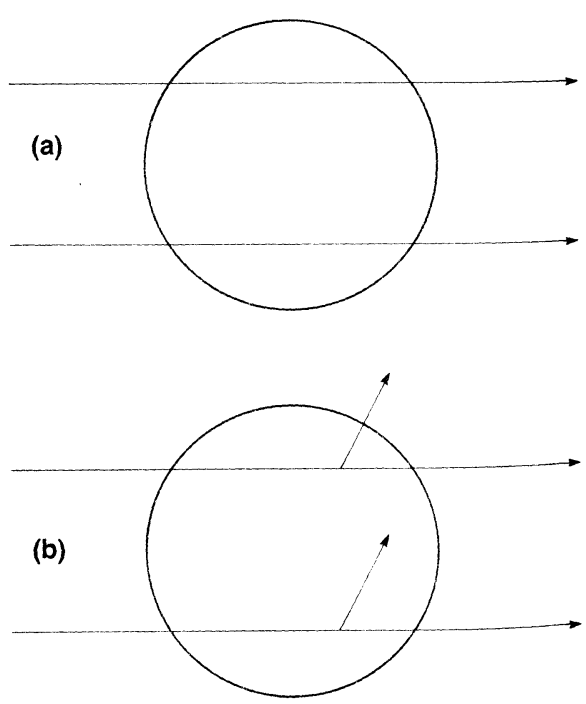

FIG. 7. Schematic representation of straggling and delta-ray effects for two identical particles of equal energy intercepting equal chord lengths in the sensitive volume. See text for explanation. 


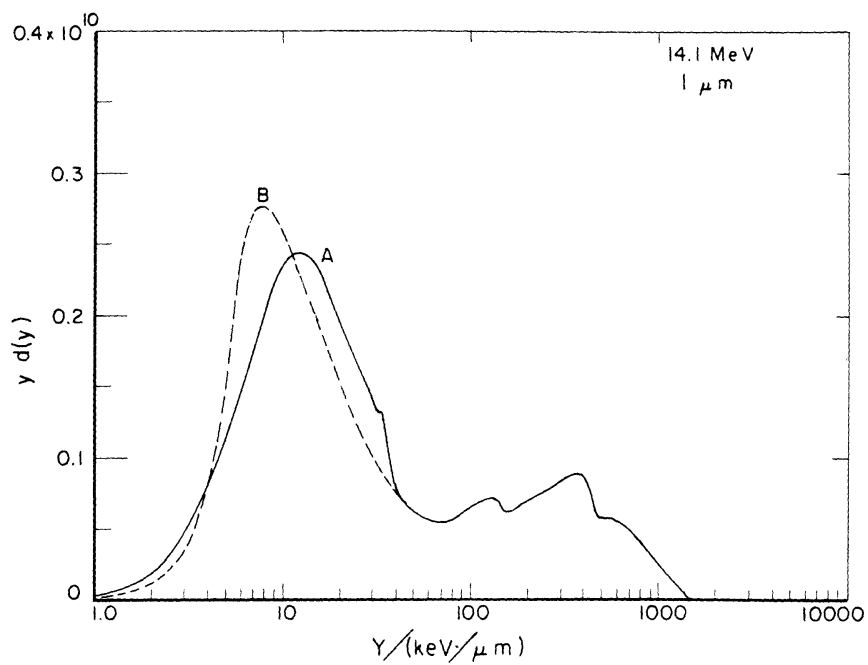

Fig. 8. Calculated microdosimetric spectra for $14.1-\mathrm{MeV}$ neutrons, $1 \mu \mathrm{m}$ diameter site. Curve $B$ is a CSDA calculation, whereas curve $A$ uses a triangular straggling distribution for the protons.

CSDA proton edge at $150 \mathrm{keV} / \mu \mathrm{m}$ is increased to around $200 \mathrm{keV} / \mu \mathrm{m}$ due to the straggling effects. In Fig. 11 is added a curve with symmetrical Gaussian straggling. This curve (dash-dot) is not far from the CSDA result, indicating why the CSDA approximation works well for cavities comparable to the range of the particles. Figure 12 shows CSDA and delta-ray approximation energy deposition curves for $14.1-\mathrm{MeV}$ for a $100 \mathrm{~nm}$ spherical site, crossers only considered. These curves are not strikingly different from the $1 \mu \mathrm{m}$ site distributions. We have also made some calculations for $10 \mathrm{~nm}$, for which the results are not fully understood. It appears that passers must be included in calculations for very small sites before we can be confident of the results.

\section{CALCULATION FOR WATER VAPOR FOR PROTONS AND NEUTRONS}

An interesting Monte Carlo calculation for protons and neutrons has recently been reported by Berger (1988) using the semi-empirical differential ionization

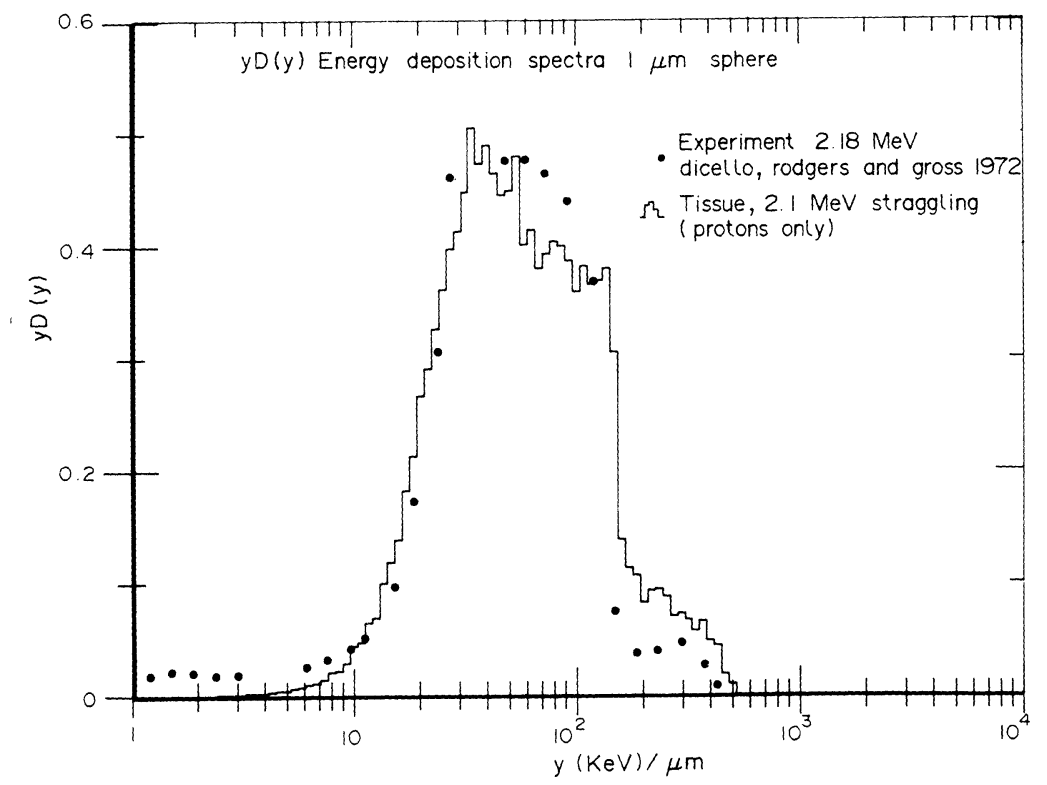

FIG. 9. Monte Carlo calculation including proton straggling for $2.1-\mathrm{MeV}$ neutrons compared to experimental result at $2.18 \mathrm{MeV}$ (Rodgers et al., 1972). 


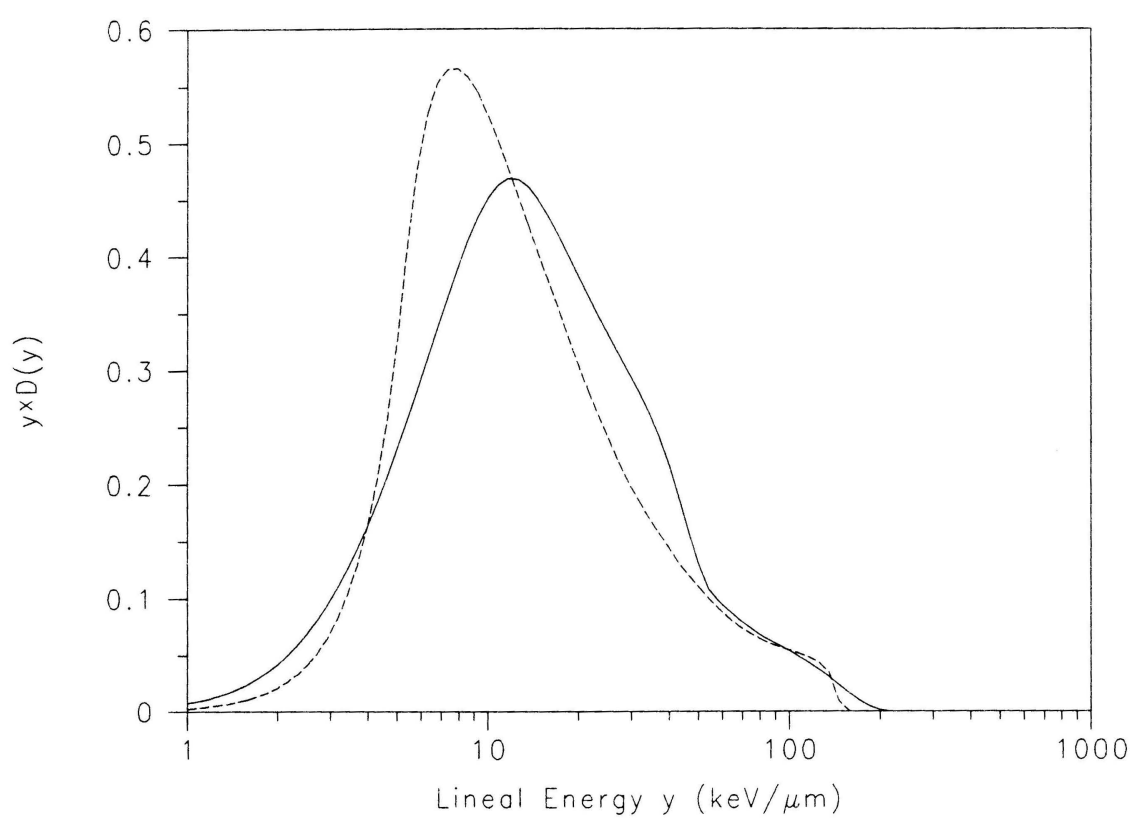

Fig. 10. Calculated microdosimetric spectra for protons from $14.1-\mathrm{MeV}$ neutrons, $1 \mu \mathrm{m}$ cavity diameter. Dashed curve is CSDA, solid curve uses the lognormal distributions of Wilson et al. (1988) including straggling and delta-ray effects. Distributions were slightly modified to give energy balance for a single track.

cross section for water vapor of Wilson and Miller (1984), including both proton energy loss straggling and transport of energy by secondary electrons. In these calculations the protons are assumed to have constant inelastic scattering cross sections along the path. In Fig. 13 Berger compares his complete calculations to the CSDA approximation for spheres of $200 \mathrm{~nm}, 400 \mathrm{~nm}, 1 \mu \mathrm{m}$, and $2 \mu \mathrm{m}$ diameter irradiated by a broad $10-\mathrm{MeV}$ proton beam. As the sphere diameter becomes smaller, the differences between the complete and the CSDA calculation increase markedly. Berger then treated the protons generated by neutrons by the quite good assumption of a flat initial spectrum of protons from zero to the neutron energy. His results for $14-\mathrm{MeV}$ and $20-\mathrm{MeV}$ neutrons are shown in Fig. 14, a linear plot in the upper

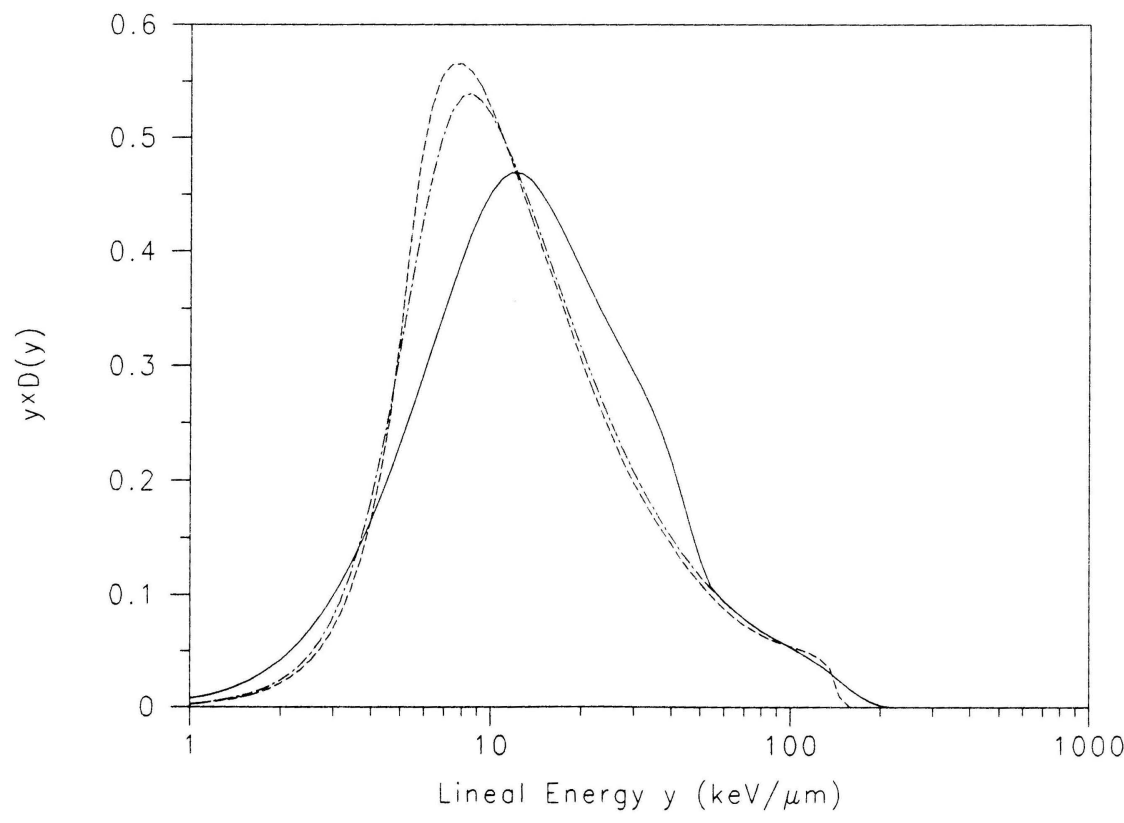

Fig. 11. Same as Fig. 10 except a curve for symmetrical Gaussian straggling has been added. Note that the Gaussian result is close to the CSDA result. 


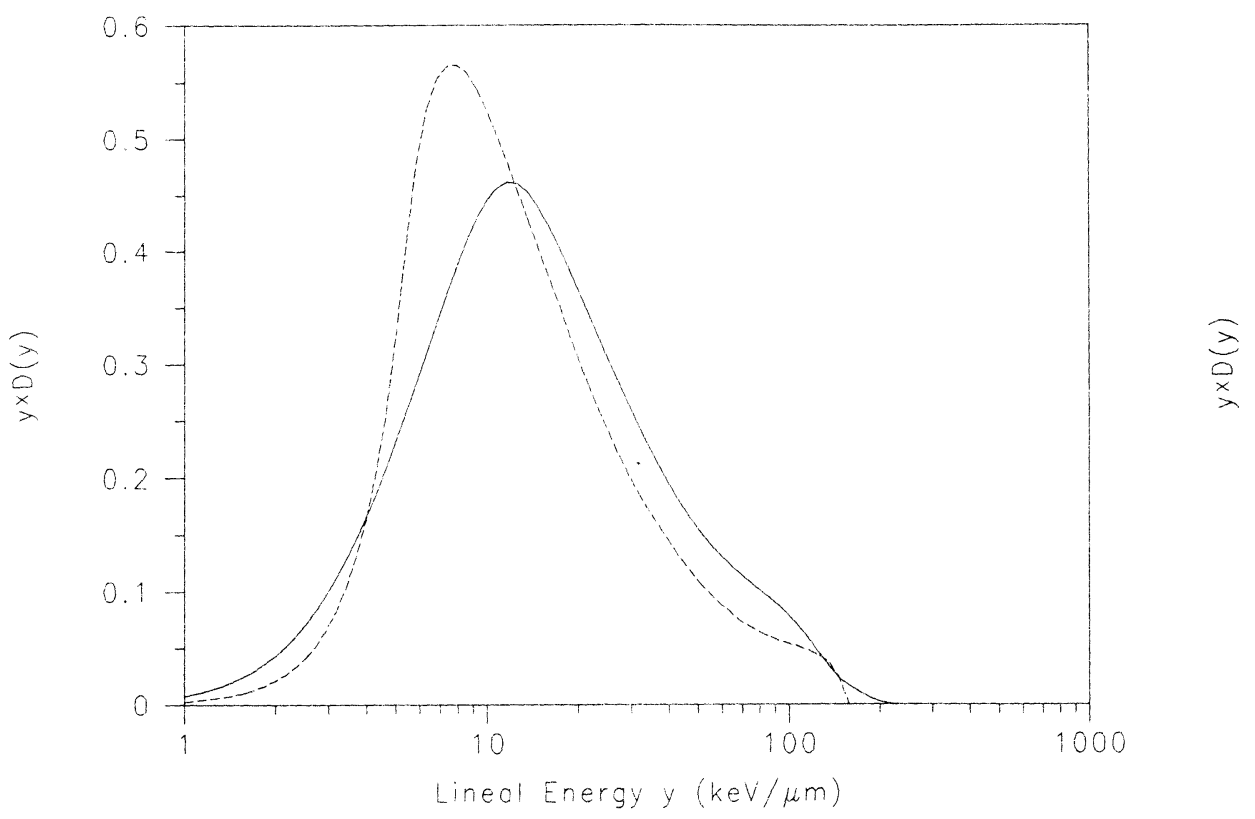

FIG. 12. CSDA (dashed) and delta-ray approximation (solid) microdosimetric spectra for protons from 14.1-MeV neutrons, $100 \mathrm{~nm}$ cavity diameter.
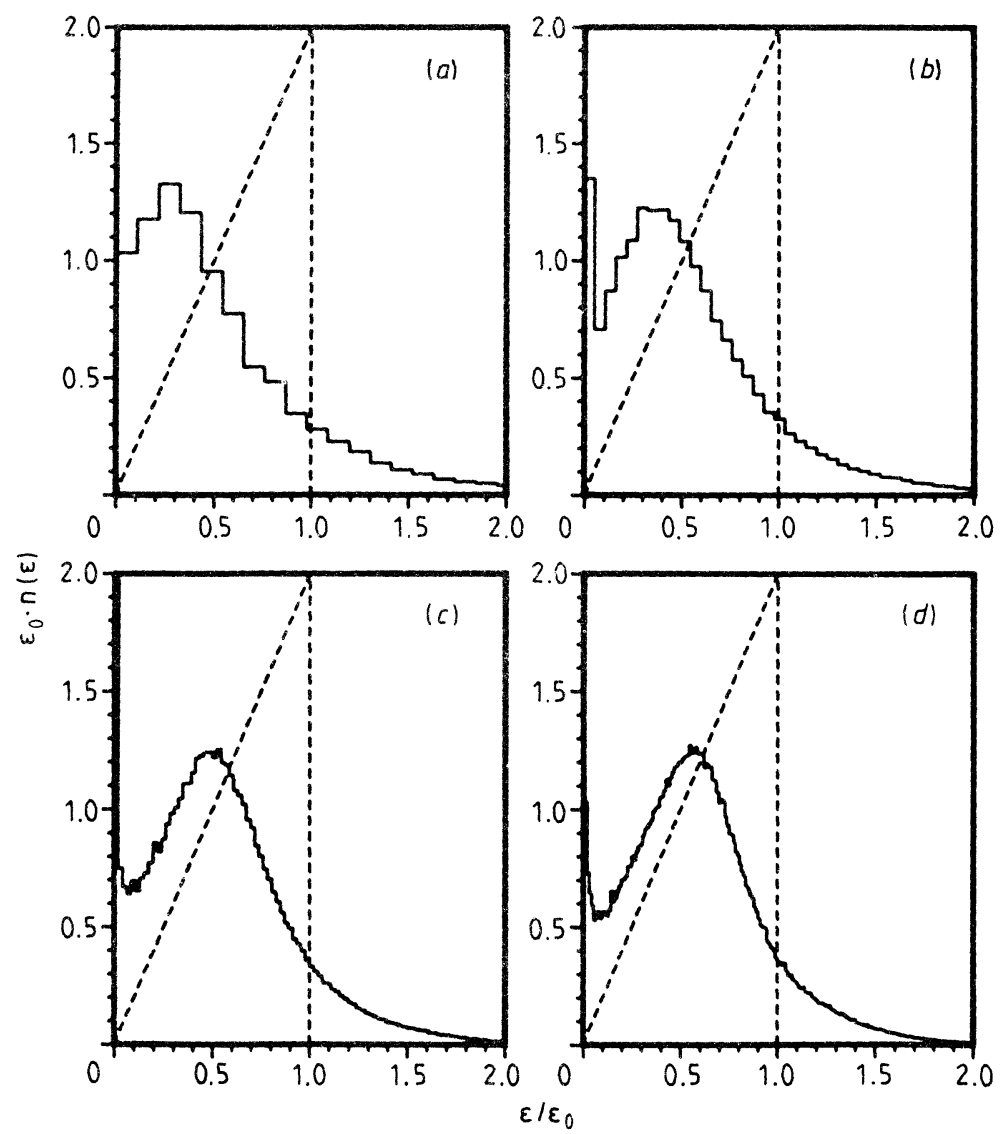

FIG. 13. Energy deposition distributions for a broad $10-\mathrm{MeV}$ proton beam irradiating spheres in water vapor with diameters (a) $200 \mathrm{~nm}$, (b) $400 \mathrm{~nm}$, (c) $1 \mu \mathrm{m}$, and (d) $2 \mu \mathrm{m}$, from Berger (1988). Monte Carlo results (histograms) include effects of both straggling and delta rays. Triangle is the CSDA result. 

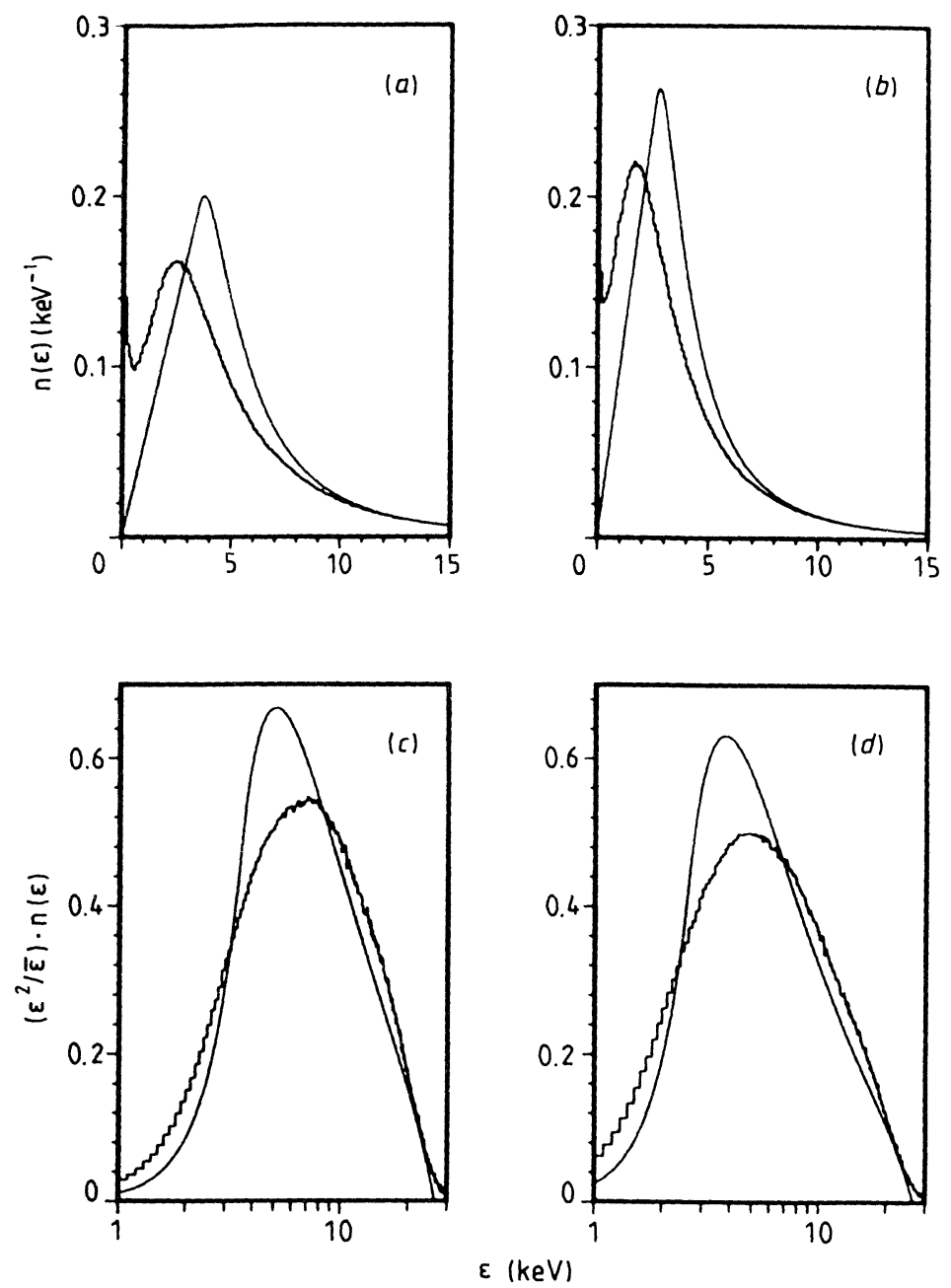

FIg. 14. Energy deposition distributions for a $1 \mu \mathrm{m}$ diameter sphere for protons from $14-\mathrm{MeV}$ neutrons (left) and 20-MeV neutrons (right), from Berger (1988). Upper curves (a) and (b) are linear, lower curves are the usual microdosimetric representation.

drawing and the usual semi-logarithmic display in the lower drawing.

\section{SUMMARY}

Track structure physics plays a major role in neutron microdosimetry and nanodosimetry. Although in some cases one can obtain satisfactory results by considering only stopping power (CSDA), as cavities become smaller and particle energies higher, it becomes increasingly necessary to consider straggling and delta rays. Much work remains to be done to include effects of passers, to consider more fully alphas and heavy charged particles, to consider cylindrical and other target shapes, and to produce detailed calculational results for a wide variety of neutron energies and sensitive volumes.

Acknowledgements-Supported in part by the Office of Health and Environmental Research, U.S. Department of Energy. Contribution of the National Institute of Standards and Technology. Not subject to copyright.

\section{REFERENCES}

Barendsen G. W. and Broerse J. J. (1966) Dependence of the oxygen effect on the energy of fast neutrons. Nature 212, 722-724.

Barendsen G. W. and Broerse J. J. (1968) Measurements of relative biological effectiveness and oxygen enhancement ratio of fast neutrons of different energies. In Biophysical Aspects of Radiation Quality, International Atomic Energy Agency, Vienna.

Berger M. J. (1988) Microdosimetric event size distributions in small sites in water vapour irradiated by protons. Phys. Med. Biol. 33, 583-595.

Caswell R. S. and Coyne J. J. (1972) Interaction of neutrons and secondary charged particles with tissue: secondary particle spectra. Radiat. Res. 52, 448-470.

Caswell R. S. and Coyne J. J. (1976) Microdosimetri spectra and parameters of fast neutrons. In Proceed ings of the Fifth Symposium on Microdosimetry, EU1 5452, pp. 97-123, Commission of The Europea Communities, Luxembourg.

Caswell R. S., Coyne J. J., Gerstenberg H. M. and Schwarh R. B. (1986) Refinement of neutron energy depositios calculations. In Fast Neutron Physics, Ruder Boskovic Institute, Zagreb, pp. 122-134. 
Coyne J. J., McDonald J. C., Menzel H.-G. and Schuhmacher H. (1981) Detailed intercomparison of calculated and measured ionization yield spectra for $20 \mathrm{MeV}$ neutrons and the implications for high energy neutron dosimetry. In Proceedings of the Fourth Symposium on Neutron Dosimetry, EUR 7448, pp. 213-223. Goodhead D. T. (1982) An assessment of the role of microdosimetry in radiobiology. Radiat. Res. 91, 45-76.

ICRU (1983). Microdosimetry. International Commission on Radiation Units and Measurements, Bethesda, Maryland, Report 36.

Katz R. and Sharma S. C. (1973) Response of cells to fast neutrons, stopped pions, and heavy ion beams. Nucl. Instrum. Meth. 111, 93-116.
Rodgers R., Dicello J. F. and Gross W. (1972) Event distribution from monoenergetic neutrons. U.S. Atomic Energy Commission Report COO-3243-1, p. 52 .

Rossi H. H. (1985) Microdosimetry and Radiobiology. Radiat. Prot. Dosim. 13, 259-265.

Wilson W. E., Metting N. F. and Paretzke H. G. (1988) Microdosimetric aspects of 0.3 to $20 \mathrm{MeV}$ proton tracks, I. Crossers. Radiat. Res. 115, 389-402.

Wilson W. E. and Miller J. H. (1984) An algorithm for secondary electron emission from water vapor by high velocity ions. Pacific Northwest Laboratory, Richland, Washington, Report PNL-SA-11768. 